\title{
The value of a skeptical approach to neurosciences in psychiatric training and practice
}

\author{
Alexandru Traicu, MD; Ridha Joober, MD, PhD
}

In teaching psychiatry, we have turned from psychological, subjective, and occasionally fantastical psychoanalytical models of illness to scientifically "harder" explanations derived from our expanding knowledge of neurobiology. Although the promises of neuroscience to root mental disease diagnoses in biology have yet to be fulfilled, the discipline has become established as the pre-eminent paradigm in psychiatric residency programs. Given this dominant role, the manner in which neuroscience is taught matters greatly in shaping the philosophical outlook and clinical practice of multiple generations of new clinicians.

As teachers and students of psychiatry, we need to remain aware of the foundational limitations of neuroscience, while appreciating the scientifically grounded framework it offers. This will foster a conservative approach in introducing neuroscience - one that is realistically proud of its achievements, while modest about its limitations. We briefly highlight some of the assumptions and limitations of neuroscience, with their implications for psychiatry, to underscore the potential value of the skeptical approach discussed in this editorial.

\section{How tenable are the assumptions of neuroscience?}

With neuroscience we have embarked on a journey of discovery with a stated goal (understanding the mind and its illnesses) and a stated craft (the study of the brain), but with an often unstated assumption: that our craft can take us there (i.e., that the mind and its illnesses can be explained through the empirical study of the brain). This is the core reductionist assumption of neuroscience. It has at least 2 aspects, the first being that all mental phenomena are ultimately reducible to physical events in the body. Another aspect is that the same general methods that have enabled the tremendous progress in the physical sciences since the seventeenth century (empiricism) will prove useful in understanding mental phenomena. This central assumption seems promising, perhaps even plausible, but remains untested. Beyond this broad core tenet, there are some additional assumptions.
One assumption is that the relatively basic levels at which we study the brain are useful in understanding high-level mental phenomena. It remains possible, however, that the levels presently accessible to neuroscientific analysis are too far removed from the psychiatric phenomena. By way of analogy, chemical reactions too can be conceptualized at various levels, from the macroscopic to the molecular, the atomic, and so on down to the most basic division, say quarks. Yet imagine the travails of the industrial chemist who should try to synthesize acetylsalicylic acid (ASA) by conducting her analysis at the level of the quarks.

Another assumption is that mental phenomena are discrete entities that map to activations in discrete brain regions and networks. But mental phenomena, such as attention, will and love, are likely holistic functions of the whole person and brain and do not reconcile easily with this modular view. Furthermore, many mental constructs have no meaningful existence in isolation; for instance, attention is so inextricably linked to arousal or motivation that it may not be truly amenable to separate study.

Yet another assumption is that indirect readings measure what they purport to measure. We have no direct access to either the mind or to the neural circuitry presumably underlying it. Rather, we must see both of them "through a glass, darkly." To study attention, we operationalize it through one facet, such as sustained attention, and then only through a behavioural proxy, such as performance on a continuous performance test, and even there we analyze a subaspect, such as omission errors. Similarly, we index brain activity through energy consumption, which we estimate through changes in glucose, blood flow, or oxygenation signals. These successive approximations risk getting further and further removed from what they purport to measure, as illustrated by a parable attributed to Einstein: ${ }^{1}$

\footnotetext{
"What's milk?," a blind man asked. "Milk's a white liquid," Einstein said. "What's white?," the blind man then asked. "White is the colour of swans," replied Einstein. "But what's a swan?," the man asked again. "It's a bird with a crooked neck," Einstein replied. "What's a crooked neck?" Exasperated, Einstein bent his arm at the elbow and replied, "Feel this; it's like a crooked neck." "Ahhh," said the blind man, "now I know what milk is."
} 
We also assume that mental phenomena are independent of the measurement set up (e.g., that what we see when we put a person in a scanner is similar to what we would see if the experimental setup was imperceptible). But when dealing with human beings, the mere act of observing possibly changes what is being observed.

Finally, yet another assumption is that DSM diagnoses reflect biologically discrete entities with distinct underlying brain alterations. However, there are concerns over the validity of symptoms-based DSM diagnoses and their appropriateness for neuroscientific research, which has prompted the National Institute of Mental Health Research Domain Criteria project. ${ }^{2}$ Although this might improve future research, much past and ongoing work uses the DSM, despite its limitations.

These issues are old and complex, touching on fields of inquiry, such as epistemology, the tension between humanities and natural sciences, and the mind-body problem, with a rich philosophical literature ${ }^{3}$ including literature regarding psychiatry. ${ }^{4,5}$ We highlighted just a few key points, not as a dismissal of neuroscientific methods in psychiatry, but rather to show that presently we are too early in our journey of discovery to safely assume that through it we will reach our goal of understanding the mind and its illnesses, hence the need for skeptical distance.

\section{What do the limitations of neuroscience mean to psychiatry?}

Currently, the most straightforward answer to the question of how the mind emerges from the brain is "we do not know." This should sound a note of caution, for it is venturesome to think that we will get very far in understanding the pathology of the mind (mental disorders) without understanding its normal workings. It is probably the case that the most straightforward answer to the question, "How and why does one develop a mental disorder?" may be equally agnostic.

But absence of sufficiently clear knowledge entails absence of expertise. In other words, whereas mental health professionals and researchers may be experts in the diagnosis and management of mental disorders, it is difficult to accept anyone's expertise in their etiopathology, given the shallowness of current knowledge compared with the profundity required for true expertise. That is not to imply that a psychiatrist or neuroscientist knows as little as the layman. However, no matter how much higher one bird flies than another, if the goal is to reach the moon, it seems unfair to call either one of them an astronaut.

Therefore, when it comes to understanding the mind and how its disorders arise we are all still beginners - both trainers and trainees. We do not mean this pessimistically. The mind of the beginner remains open to discovery and has not prematurely grasped too poignantly notions that, at best, can only hope not to be too wrong. However, openness and curiosity naturally seek explanations. Therein lay both the attraction and the peril of neuroscience for psychiatry, for in our eagerness to understand we may become attached to misunderstandings, either singly, as a private conviction, or as groups prey to a fad.
The siren song of neuroscience holds particular charm for psychiatry residents. Psychiatric training skimps on neuroscience, and the training that is offered focuses on abnormal findings without solid grounding in the fundamentals of neuroscience, let alone in the methodological or epistemological foundations of the field; this is a problem that the National Neuroscience Curriculum Initiative (www.nncionline .org/) may help remedy. On the other hand, constant work with fascinating mental phenomena among colleagues from fields of medicine whose biology is better understood naturally makes one reach for neurobiological explanations. This can lead to a state of half-knowledgeable avidity that reflexively welcomes new discoveries as potentially useful, or at least illuminating, without actively reflecting on the likelihood of their truthfulness.

\section{How could skepticism help?}

To avoid such a pitfall and to maintain the openness of a beginner's mind, we propose that the teaching and practice of skepticism would be of some use. We are not referring to an ideological or dismissive type of skepticism that instinctually denigrates new findings as insufficient (what new finding in the study of the mind would ever be sufficient in and of itself?) or places undue burdens of proof. Even less do we advocate for excessive fault-finding in each study. It is not enough (or even of primary importance) to think critically of individual facts, articles, or analyses, for one may still be carried by the torrent of research flowing from whichever model is in common favour.

What seems more important is developing and maintaining an attitude of humility and detachment. This includes maintaining awareness of the limitations of our models and of their underlying untested assumptions, keeping a critical distance not only from new things we learn, but also from what we think we already know, and, perhaps most importantly, not taking ourselves and our knowledge very seriously, for future generations will likely shake their heads with bemusement at some of our concepts.

Models are indispensable in clinical practice. At their best, they function like the masks of classical Greek theatre, allowing us to assume whatever role seems most helpful. When faced with a patient's agitation, we might look at it as neuroscientists, conceptualizing it as amygdalar overactivation. In the absence of progress, we change masks and try a behaviourist or psychodynamic one instead. The key here is the ability to put on, but also to take off the masks. But should we believe a model too unquestioningly, the mask will adhere so tightly as to grow roots into our flesh: we will identify with our role and lose the useful flexibility of other perspectives. Skepticism can be conceptualized as both the space between the mask and the face (critical distance) and as the clear knowledge that we are only wearing the mask of "expert on the mind," rather than being such. In other words, it may help to be skeptical not only of colleagues, but also of ourselves.

We propose a few ways, which can be taught through practice, to foster an attitude of healthy skepticism: 
- asking upon learning of some new finding, "How is this wrong?" rather than "How is this applicable to my practice?";

- not allowing oneself to be intimidated by mathematically complex analyses (they allow more scope for errors in thinking, all the while making them harder to detect);

- answering patients' inquiries regarding how their illness came about, how it relates to their brains, or how medications work, with utmost honesty (in most cases this will probably mean saying "I don't really know");

- trying to encounter each patient with fresh curiosity unencumbered by an arsenal of ready-made mechanistic explanations (e.g., serotonin deficit, overactive amygdala);

- remembering that currently the most direct avenue to the inner experience of a patient remains experiential (either through patients' self-analysis and phenomenological report, or through empathy on our part) rather than empirical;

- emphasizing at both the personal and program level the areas currently neglected by residency training, including the history of psychiatry, epistemology, and the nature of the mind, to develop a framework within which neuroscientific discoveries can be integrated; and

- ensuring at the training program level that sufficient grounding in the fundamentals of neuroscience is achieved before introducing pathological findings in various disorders.

Skeptical awareness of neuroscience's assumptions may help both us and our trainees notice the contradictions and paradoxes in published research. Not imagining oneself an expert in how patients came to suffer the way they do will also foster humility and carefulness in practice; after all, we tend to tread more lightly when we know we are walking on thin ice.

Acknowledgements: The authors thank Dr. Vasavan Nair for his invaluable help in writing this manuscript.

Affiliations: From the Douglas Institute and the Department of Psychiatry, McGill University, Montreal, Que., Canada.

Competing interests: R. Joober sits on the advisory boards and speakers' bureaus of Pfizer, Janssen Ortho, BMS, Sunovion, Otsuka, Lundbeck, Perdue and Myelin; he has received grant funding from them and from AstraZeneca and HLS. He has received honoraria from Janssen Canada, Shire, Lundbeck, Otsuka, Pfizer and Perdue for CME presentations and royalties from Henry Stewart talks. None declared by A. Traicu.

\section{References}

1. O'Donnell M. The Barefaced Doctor. Leicester (UK): Matador; 2013.

2. Casey BJ, Craddock N, Cuthbert BN, et al. DSM-5 and RDoC: progress in psychiatry research? Nat Rev Neurosci 2013;14:810-4.

3. Gadamer HG. Truth and method 2nd rev. ed. New York (NY): Crossroad; 1989.

4. Kandel ER. A new intellectual framework for psychiatry. Am J Psychiatry 1998;155:457-69.

5. Kendler KS. Toward a philosophical structure for psychiatry. Am J Psychiatry 2005;162:433-40.

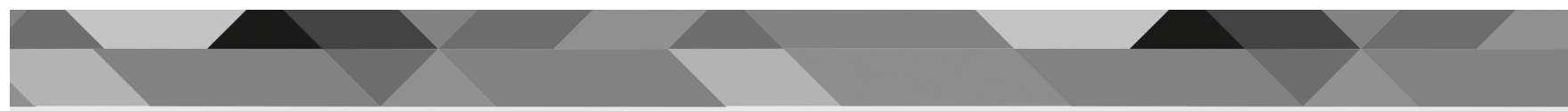

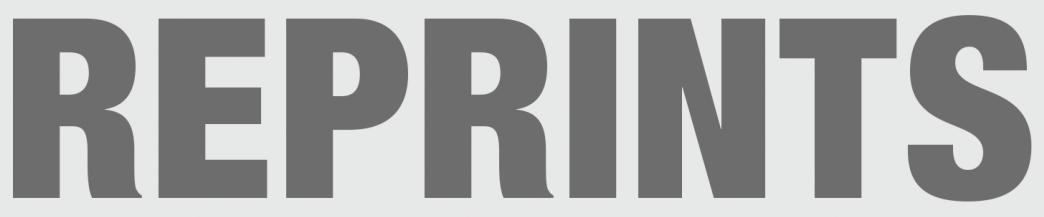

from the CMAJ Group
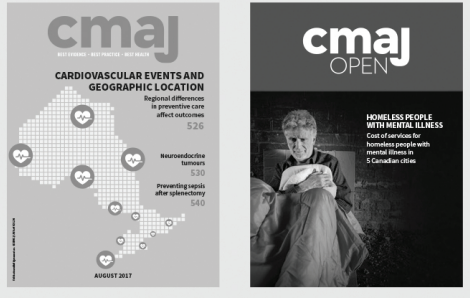

Going to a conference? Teaching a course?

Need extra copies?

Contact Sheridan Press

1-800-635-7181 ext.8065 | marcus.glover@sheridan.com www.sheridan.com/cma/eoc

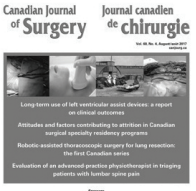

$-$

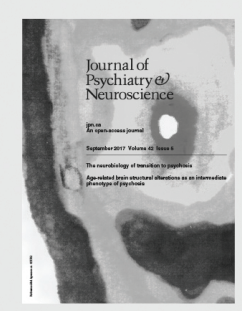

